\title{
Laser-Induced Fabrication of a Transsubstrate Microelectrode Array and Its Neurophysiological Performance
}

\author{
SUNG J. KIM, MEMBER, IEEE, MYUNGHWAN KIM, SENIOR MEMBER, IEEE, \\ AND WILLIAM J. HEETDERKS, MEMBER, IEEE
}

\begin{abstract}
Technical problems associated with the monolithic integration of active circuitry into a high-density microelectrode array for neural recordings include passivation of the active circuit area, capacitive coupling of switching transients, and nonuniformity of the recording surface. These problems can be eliminated by implementing the passive and active elements on opposite planes of a substrate and interconnecting them with transsubstrate conductive channels.

As the first step toward this integrated active transsubstrate microelectrode array (TMEA), a passive TMEA was fabricated and is reported in this paper. An array of six transsubstrate conductive channels, each $100 \mu \mathrm{m}$ in diameter and spaced on $100 \mu \mathrm{m}$ centers, were made in a 7 mil thick silicon substrate using a laser-induced diffusion technique. The transsubstrate channels are metal doped p-type channels embedded in a n-type silicon wafer. The $p-n$ junction thus formed provides good channel-to-substrate and channel-to-channel isolation.

Excellent recordings were made from crayfish giant axons using TMEA. The signal-to-noise ratio was as large as 10 to 1 with no crosstalk observed between adjacent channels. The TMEA showed no degradation with long-term use.
\end{abstract}

\section{INTRODUCTION}

$S^{\prime}$ IMULTANEOUS recording from a multiple number of nerve cells is essential to the understanding of a nervous system since the information flow is not stored in single cells but is distributed across a population of cells. A number of methods has been used to achieve such a recording. Grouping a number of glass micropipette or thin metal wires into a small recording area is a conventional technique, but difficulties are often found in controlling the dimensions in fabrication and in manipulating individual electrodes in experiments.

Photolithographic techniques have been used in recent years to generate arrays of microelectrodes with precisely controlled dimensions. A number of "printed circuit" microelectrode arrays (MEA's) have been fabricated using these techniques in different configurations and successfully used in neurophysiological recordings [1].

More important, successful attempts have been made to make such "passive" arrays "active" by integrating buffer amplifiers into them. The major benefit of the on-chip in-

Manuscript received August 23, 1984.

S. J. Kim was with the School of Electrical Engineering, Cornell University, Ithaca, NY. He is now with AT\&T Bell Laboratories, Allentown, PA 18103.

M. Kim is with the School of Electrical Engineering, Cornell University, Ithaca, NY 14853.

W. J. Heetderks was with the School of Electrical Engineering, Cornell University, Ithaca, NY. He is now with Butterworth Hospital, Grand Rapids, MI 49506. tegration of the buffer amplifier is in the reduced electrostatic noise pickup due to the shortened lead length from a high-impedance microelectrode site to an amplifier input. Wise et al. [2] used 2 JFET (junction field effect transistor) amplifiers with their depth probe electrode for simultaneous stimulation and recording. In the monolithic integration of a planar-type MEA, Jobling et al. [3] used a part of each MOSFET (metal oxide semiconductor FET) gate metallization as an electrode site. With nine electrode sites spaced at $1 \mathrm{~mm}$, each $400 \mu \mathrm{m}^{2}$ in area, and with offchip multiplexing circuits, they obtained single unit recordings from slices of rat hippocampus in vitro.

\section{The Need for a Transsubstrate Microelectrode ArRaY}

The experiments to date have integrated passive microelectrodes and active electronic devices on a single surface of the semiconductor substrate. However, in applications of MEA where high density of electrodes and consequent multiplexing of electrode signals are required, this configuration has some critical problems.

First, the electrode surface that now contains the active devices has to be specially passivated to resist the diffusion of the sodium ions from the saline where a recording usually takes place. The diffused sodium ions cause oxide charging which degrades the performance of $p-n$ junctions and also cause a threshold voltage shift in MOS devices. Second, the switching transients from the multiplexing circuitry, being capacitively coupled through the thin passivation layer, will appear at the recording electrode sites as a major source of noise.

The uniformity of the recording surface is another concern. Since the extracellular current density decreases as an inverse square function of distance, intimate contact should be established between recording sites and neurons. Bonding wires will elevate the surface structure to make intimate contact difficult. Finally, more silicon area is required to accommodate the active circuit.

We propose, as a solution to all these problems, a structure that separates the electrodes and the active electronics on opposite planes of a substrate and connects them through an array of isolated transsubstrate conductive channels which extend through the thickness of the substrate. We name this a transsubstrate microelectrode array (TMEA). 

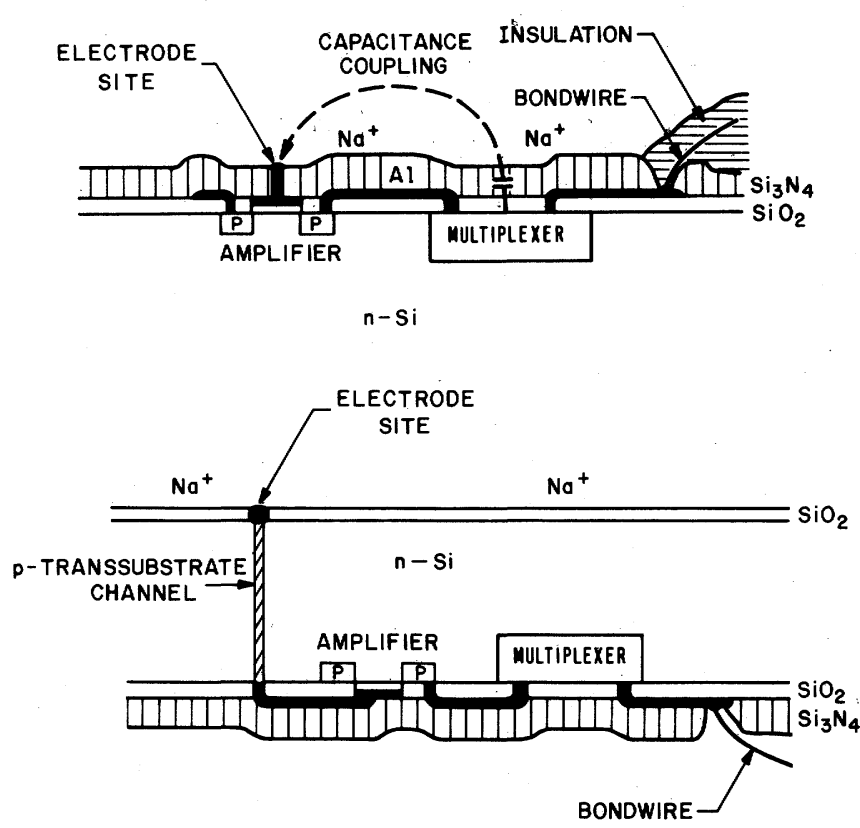

Fig. 1. Conventional and transsubstrate microelectrode arrays. (a) shows problems with conventional active microelectrode array (MEA) including diffusion of sodium ions, capacitance coupling of switching transients, and nonuniform surface. (b) shows the proposed solution by active transsubstrate microelectrode (TMEA) array.

Fig. 1(a) schematically displays the problems in a conventional active MEA while (b) shows the solution by an active TMEA. With the TMEA in (b), the silicon substrate is acting as an effective diffusion barrier to the sodium ions, and the other problems listed above become irrelevant because of the separation of active devices from recording sites.

For the rest of this paper, we will discuss the fabrication of a passive TMEA without the active circuits as the first step toward the active TMEA and show the results of electrical measurements and in vitro recordings.

\section{Laser-Induced Transsubstrate Channels}

An effective way of producing conductive channels, which are isolated from the host semiconductor substrate and from each other, is to surround them with $p-n$ junctions (i.e., build p-type channels in an n-type substrate as in Fig. 1(b) or vice versa). This way, the electrode channels are isolated from each other by two $p-n$ junction depletion region capacitances.

An unbiased cylindrical p-n junction with a $10 \mu \mathrm{m}$ diameter and a $200 \mu \mathrm{m}$ height will have a junction capacitance of only $0.46 \mathrm{pF}$. This can be compared to a shunt capacitance of $4.5 \mathrm{pF}$ in a conventional MEA using a 1 $\mu \mathrm{m}$ thick layer of photoresist as an insulation over a typical conductor which is $10 \mu \mathrm{m}$ wide and $10 \mathrm{~mm}$ long. The junction capacitance can be further reduced by applying a reverse bias to the junction. If a reverse bias is applied, however, an insulation such as phosphorus doped silicon dioxide, photoresist, or silicon nitride is required over the substrate to isolate the $p-n$ junction from the saline solution.

We used a laser deep diffusion technique to produce
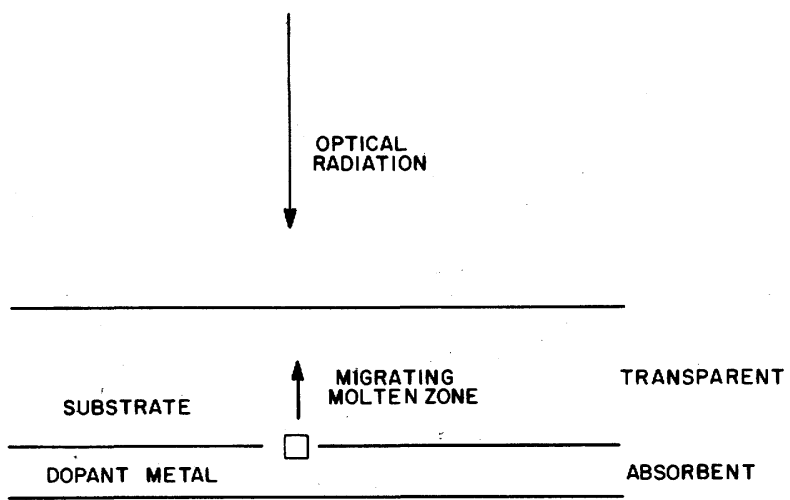

Fig. 2. Photoinduced zone migration (PIZM).

doped transsubstrate channels in a silicon substrate. The principle of the technique is illustrated in Fig. 2. A molten zone in a solid can be made to migrate through it by applying a temperature gradient across the zone, in which the solid is single crystal silicon and the molten zone is a solution of silicon and a dopant metal. By choosing a laser which is transparent to silicon, but is absorbed by the dopant metal, a molten zone is generated at the interface as the temperature exceeds the silicon-metal eutectic point. Since the zone absorbs the energy strongly at the edge facing the light, a temperature gradient is formed across the zone and it migrates toward the light source. Silicon is doped with the dopant metal in the wake of the migrating zone. This technique was invented by Kimerling et al. [4] and has been termed "photoinduced zone migration" (PIZM).

This method was chosen over other temperature gradient zone melting methods (for example, see [5]), for it offered an optically controlled resolution, shorter processing time, lower substrate temperature during processing, and more directional migration of zone.

\section{FABRICATION}

A 50 weight percent alloy of aluminum and gallium was selected as a p-type dopant metal to produce p-type channels in an n-type silicon substrate. Aluminum is suitable for film deposition and provides the least lattice mismatch with silicon. Gallium was added to lower the eutectic temperature with silicon at which the PIZM process initiated. Both aluminum and gallium migrated during the PIZM process forming aluminum-gallium doped silicon in the wake of the migration, and appeared on the surface on which the laser was incident.

As the light source, $\mathrm{CO}_{2}$ laser was chosen. It is transparent to silicon, but is absorbed by the dopant metal, as is required by PIZM. The beam was operated in the CW mode and focused into a $200 \mu \mathrm{m}$ spot using a germanium lens.

The wafers used were n-type silicon wafers of 1-10 $\Omega$. $\mathrm{cm}$ resistivity, (111) or (100) orientation, and 5-10 mil thickness. Both sides were polished to avoid the surface absorption on the side where the laser beam is incident, and to enhance interfacial uniformity between silicon and metal on the other side. 
The wafer preparation before the laser processing consisted of standard cleaning, oxidization, and metallization procedures. The metallization was patterned and was $0.5-$ $1 \mu \mathrm{m}$ thick depending on the wafer thickness. The pattern used was a matrix of squares, each 30 mils a side and separated by 10 mils from each other. Alloy treatment was not necessary because the eutectic point was lowered by using the aluminum-gallium alloy. The wafers were then scribed into rectangular dies of 400 by 900 mils for easier handling.

A die was then vacuum mounted on a micrometer with the unmetallized side facing the laser, and was aligned and exposed to the laser. The alignment was done with the metal pads on the corners serving as alignment marks. When a pad was aligned and exposed to a high-power laser, glowing started immediately. Then the die was moved by a known distance by the micrometer to align and expose a target metal pad.

To process a channel in a 7 mil thick substrate, $8 \mathrm{~W}$ of total laser power was used initially. Once the process was initiated, however, the power was reduced to $6 \mathrm{~W}$. This reduction of power during exposure was found to be very effective in reducing dislocations or cracking generated by thermal misfit between the locally heated area and the surrounding cooler area of the wafer. Each site was exposed for $40-60 \mathrm{~s}$, and the process was repeated for the other sites. The shape of a channel after the completion of PIZM is schematically shown in Fig. 3.

The wafer was again scribed into individual chips each containing a desired number (usually 6) of transsubstrate channels, which were then bonded and mounted on a glass carrier. The dopant metal pads, partly consumed by PIZM process, were also used as bond pads. Gold bond wires were extended with insulated silver wires to a multipin microconnector. The assembly was then sealed with several coatings of Silastic ${ }^{\mathrm{TM}}$ silicone rubber. Finally, the electrode sites (the emerged metal zones) were gold plated. The completed electrode is shown in Fig. 4 where (a) and (b) show the top view of the TMEA with gold-plated electrode sites, and the side view of the whole electrode assembly, respectively.

\section{Characteristics of Fabricated Channels}

\section{A. Geometry}

Fig. 5(a) shows a picture of a typical metal rich zone which emerged on the surface of silicon as a result of PIZM. The dark region in a backside picture in Fig. 5(b) represents a region where the metallization was alloyed with silicon. Near the silicon-metal interface where PIZM started, a laterally broadened junction was formed. This is because a larger area is heated above eutectic temperature near the interface than in the rest of the substrate due to the metal's larger thermal conductivity.

Although a lapping procedure could be used to remove the broadened junction tail, this was not done because we set the interelectrode spacing (40 mils) greater than the radius of the junction tail (about 20 mils).

${ }^{\mathrm{rm}}$ Silastic is a Dow Corning registered trademark

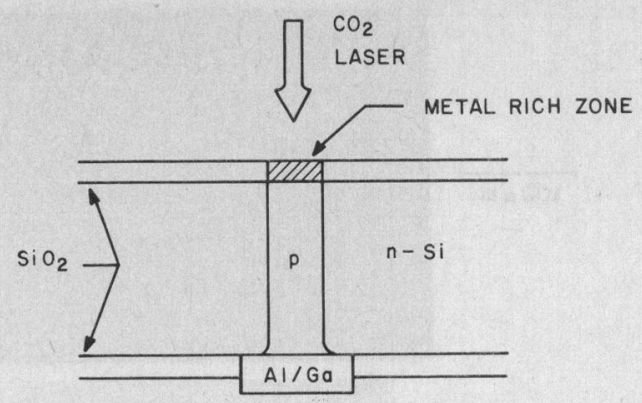

Fig. 3. Transsubstrate channel on completion of PIZM.

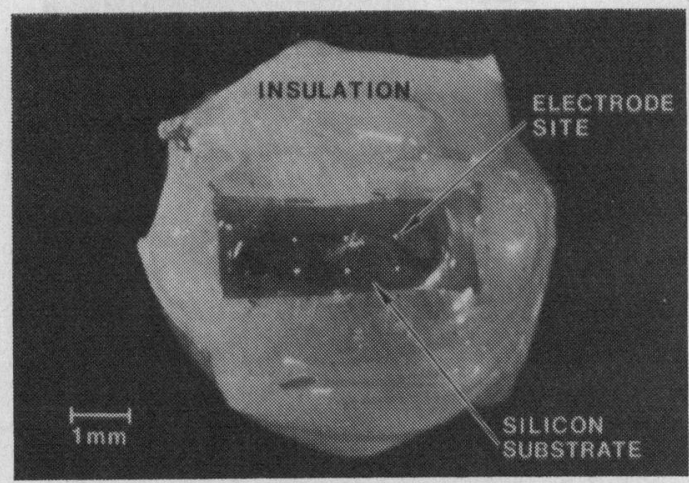

(a)

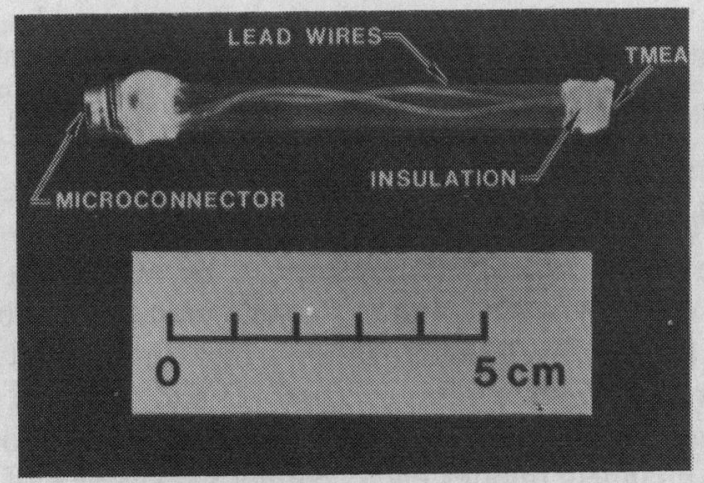

(b)

Fig. 4. Completed TMEA assembly. (a) shows top view of the TMEA, and (b) shows side view of the whole assembly.

\section{B. Junction Capacitance}

The transsubstrate $\mathrm{p}-\mathrm{n}$ junction consisted of a cylindrical junction of $100 \mu \mathrm{m}$ in diameter and 7 mils in height with a broadened tail, and a square Schottky junction at the metal-silicon interface as depicted in Fig. 5(c). Ignoring the shallow broadened tail, the junction area is estimated to $5.9 \times 10^{-3} \mathrm{~cm}^{2}$ and the corresponding junction capacitance is calculated to be $45.02 \mathrm{pF}$ with no bias in a substrate of $10 \Omega \cdot \mathrm{cm}$ resistivity.

As discussed before, a smaller junction means better isolation of the channels. For reduced junction capacitance, however, a smaller channel should be made. The minimum diameter of the channels achieved by the present technique, using a $\mathrm{CO}_{2}$ laser, is $100 \mu \mathrm{m}$. Alternative techniques to achieve smaller channel sizes are discussed later in Section VII. 
(a)

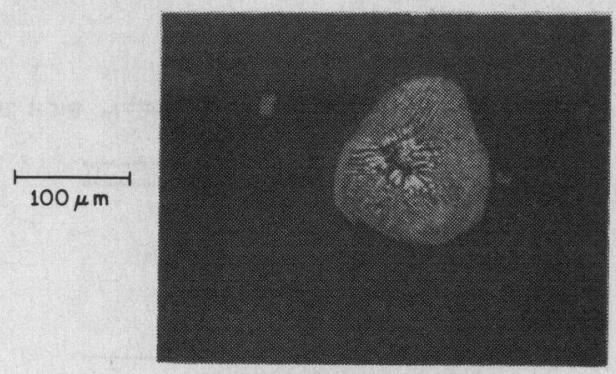

(b)

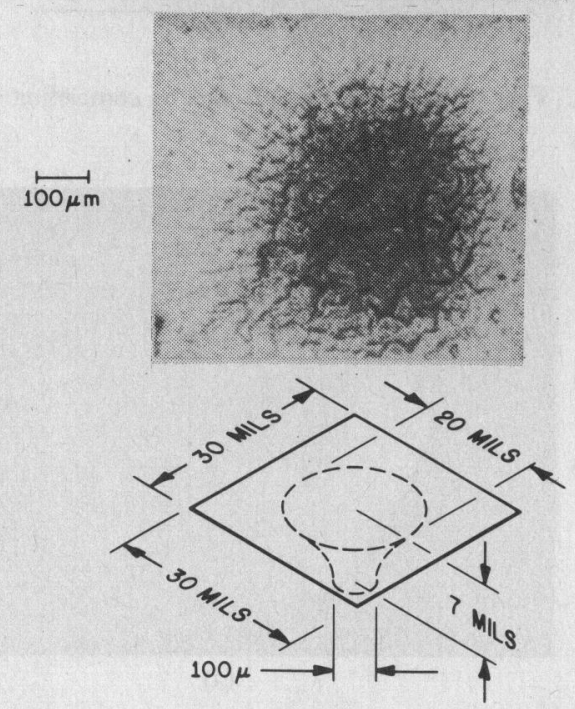

(c)

Fig. 5. Transsubstrate junction. (a) is a picture of the metal rich zone emerged on the front surface of the substrate, (b) is a picture of the back surface showing the alloyed metallization, and (c) shows schematically the shape of a transsubstrate $\mathrm{p}-\mathrm{n}$ junction.

\section{Channel Conductance}

The conductance was determined by measuring resistance of two transsubstrate channels made in a substrate using a broad-back metallization which shorts the two together. Fig. 6 shows this method (a) and a typical result (b) showing about $1.25 \Omega$ of resistance. Equal geometry of the channels implies each channel's resistance of 0.625 $\Omega$, which then can be converted to an average doping density of about $0.5 \times 10^{19} \mathrm{~cm}^{-3}$ of this cylindrical p-type channel of $100 \mu \mathrm{m}$ diameter and $7 \mathrm{mil}$ height. The resistance of the channel was independent of the junction bias voltage.

\section{Saline-Electrode Impedance}

Experimentally, the metal electrode-saline interface acts as a nonideal capacitance with phase angle relatively independent of frequency. Typical values of the amplitude and the phase of the saline-electrode impedance of the gold-plated electrode sites in the TMEA are plotted in Fig. 7. Before gold plating, the area of the electrode site was measured as about $8 \times 10^{-5} \mathrm{~cm}^{2}$. The impedance is $110 \mathrm{k} \Omega$ at $1 \mathrm{kHz}$, and its frequency dependency over the bandwidth can be approximated as $f^{-0.68}$ with phase of about $73^{\circ}$. Therefore, the electrode interface in the TMEA behaved as a capacitance of $1.45 \times 10^{-9} \mathrm{~F}$ (or $18 \mu \mathrm{F} /$ $\mathrm{cm}^{2}$ ). These values are similar to the ones reported in [2] and [3].

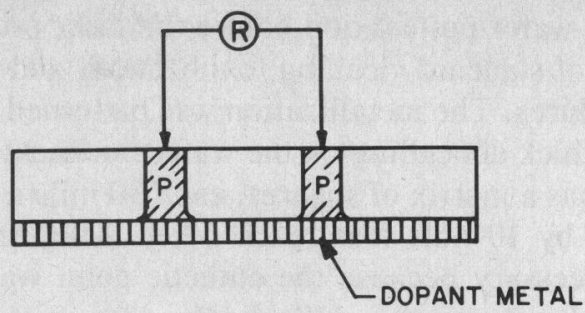

(a)

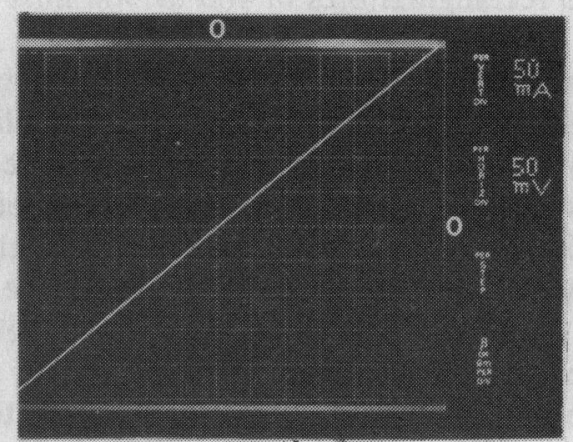

(b)

Fig. 6. Measurement of doping density of transsubstrate channel. (a) shows the method, and (b) a typical result.

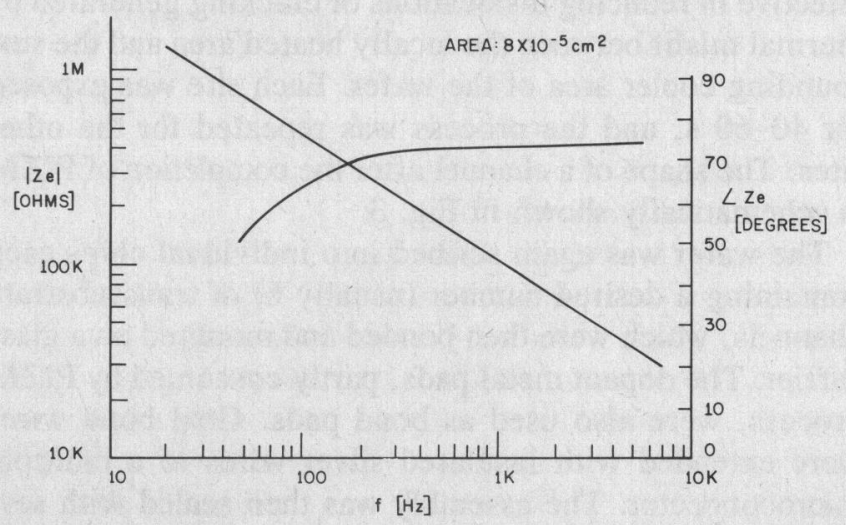

Fig. 7. Amplitude and phase of the gold-plated electrode impedance with respect to frequency.

\section{E. $p-n$ Junction Characteristics}

The reverse and the forward characteristics of the transsubstrate $p-n$ junction made on a substrate of $10 \Omega \cdot \mathrm{cm}$ are shown in Fig. 8(a) and (b), respectively. The $I-V$ characteristics can be described by

$$
J=J_{s} \exp (q V / n k T) \quad(\text { for } V<0.3 \mathrm{~V})
$$

where

$$
\begin{aligned}
J= & \text { current density in amperes per } \mathrm{cm}^{2}, \\
J_{s}= & \text { saturation current density }=1.7 \times 10^{-6} \text { amperes } \\
& \text { per } \mathrm{cm}^{2} \\
n= & \text { ideality factor }=1.67,
\end{aligned}
$$

and $k T / q=0.0259 \mathrm{~V}$.

These characteristics are typical of silicon $\mathrm{p}-\mathrm{n}$ junction diodes except for the reverse current which increases rapidly with the increase of reverse bias voltage leading to the soft breakdown. This is due to the misfit dislocation generated by thermal stress during PIZM. The dark lines in the surface pictures of Fig. 9 show the dislocation net- 


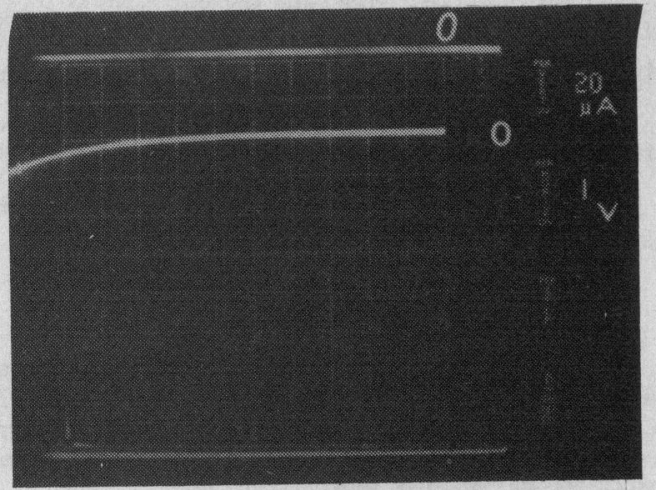

(a)

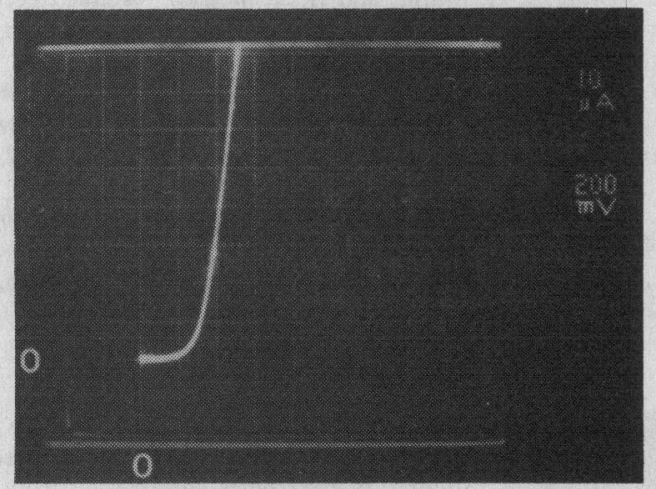

(b)

Fig. 8. Transsubstrate $\mathrm{p}-\mathrm{n}$ junction $I-V$ characteristics. (a) Reverse and (b) forward. The junction was made on a $10 \Omega \cdot \mathrm{cm}$ substrate.

work surrounding the emerged metal zone shown as the white area at the center. The wafer was in (100) orientation, and a staining solution (HF solution with 0.5 percent $\mathrm{HNO}_{3}$ ) was used to reveal the dislocation network.

\section{RECORDINGS}

An extracellular in vitro testing of the TMEA was done using the medial giant nerve of a crayfish (Procambarus clarkii). The nerve is a mixture of motor axons and sensory axons, and is large in size $(200-450 \mu \mathrm{m}$ in diameter). It is spontaneously active but can also be easily stimulated by softly rubbing the tail.

A TMEA with 6 electrode sites was mounted at the bottom plane of a recording chamber with the recording surface facing up. Then the nerve was laid over the array along its center line as shown in Fig. 10. The nerve used was dissected away from the abdomen to facilitate the recording, but left intact in the animal's tail in order to prolong its viability and to permit it to be stimulated.

A typical 6-channel recording by a TMEA is displayed in Fig. 11. The nerve was not stimulated. The signals recorded reached $40-150 \mu \mathrm{V}$ peak to peak while the noise was about $15 \mu \mathrm{V}$. This oscillograph was made by successively recording potentials from each of six electrodes.

Good interchannel isolation of the TMEA is demonstrated in a simultaneous recording shown in Fig. 12(a). An action potential was recorded by three equally spaced (40 mil) electrode sites (channels $A, C$, and $E$ in Fig. 10) that were linearly placed under the nerve. The same time delay exists between the occurrence of action potentials

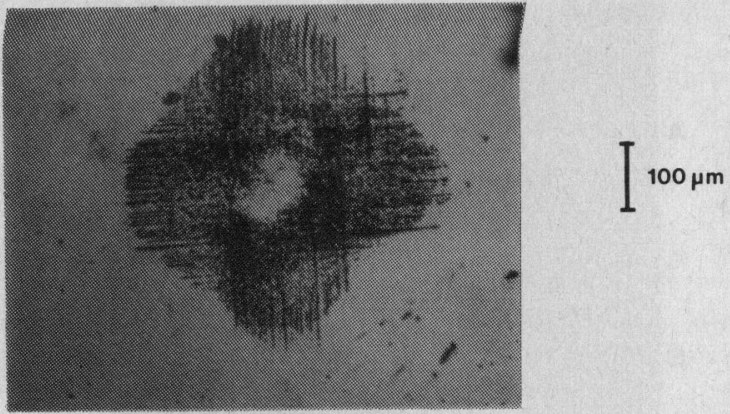

Fig. 9. Stained misfit dislocation network (shown as dark lines) surrounding the emerged metal zone (shown as white area at the center) on the surface of a (100) wafer.

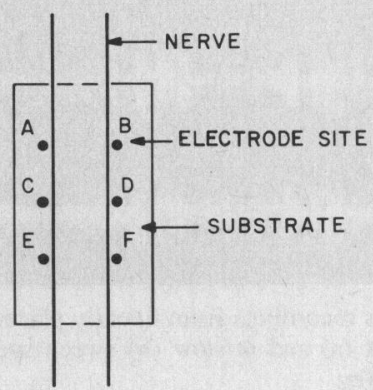

Fig. 10. Nerve laid over the TMEA. $A-F$ denote each of 6 electrode sites.

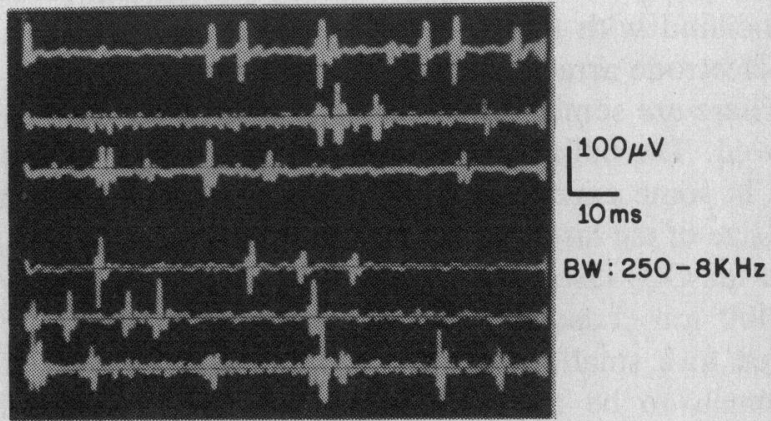

Fig. 11. Successive recordings taken from 6 electrode sites. Recording bandwidth was $250-8 \mathrm{kHz}$.

in traces $A$ and $C$, and in $C$ and $E$. This represents the delay caused by the action potential traveling over the interelectrode spacing. The conduction velocity of this unit calculated from the oscillograph is about $10 \mathrm{~m} / \mathrm{s}$. No crosstalk is observed among the traces. Fig. 12(b) shows the same recording at slower sweep speed.

The TMEA used for the recordings above did not show any signs of degradation after repeated use for a total of $40 \mathrm{~h}$.

\section{SUMMARY AND DISCUSSION}

The objective of making a transsubstrate microelectrode array has been achieved. An array of transsubstrate conductive channels has been produced and tested as a passive electrode array. The ability of the array to record and transmit multichannel neural signals has been demonstrated both in electrical measurements and neurophysiological experiments. The uniqueness of this device lies in the fact that it allows the active and the passive 
(a)

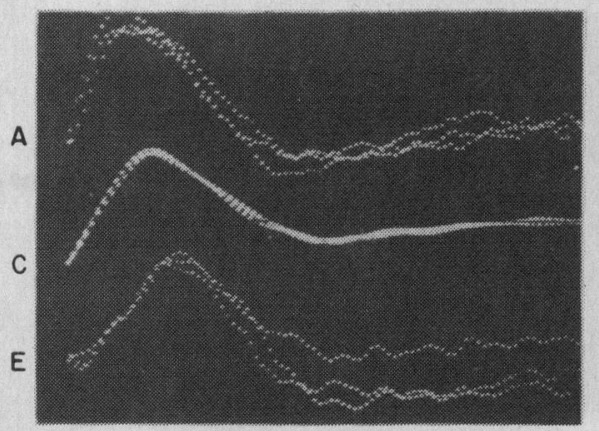

(b)

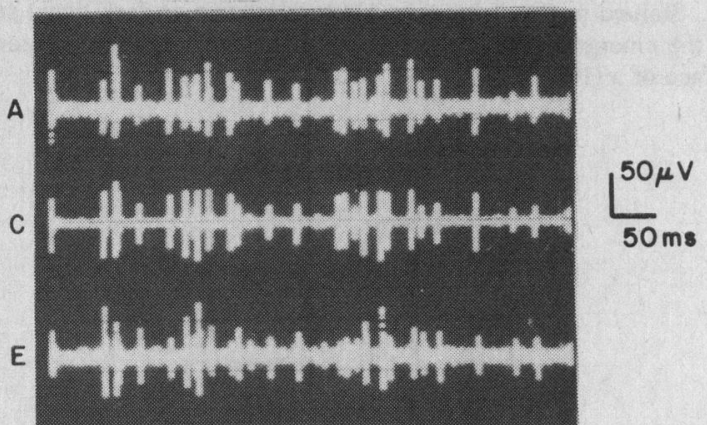

$\frac{L^{20 \mu v}}{0.2 \mathrm{~ms}}$

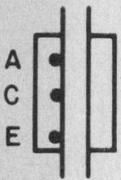

$0 \mathrm{~ms}$

Fig. 12. Simultaneous recordings from linearly placed channels $(A, C$, and

$E$ ) displayed at fast (a) and at slow (b) sweep speed. Recording bandwidth was $250-8 \mathrm{kHz}$.

elements of the electrode array to be implemented on opposite planes of a substrate, thereby eliminating problems associated with the monolithic integration of active microelectrode arrays.

There are some aspects of the device which may be improved. The bulkiness is one problem that may hinder its use in some experiments. The channel size is limited by the size of the laser beam. The typical minimum focal spot size for $\mathrm{CO}_{2}$ laser of $10.6 \mu \mathrm{m}$ wavelength is on the order of $100 \mu \mathrm{m}$. Lasers with shorter wavelengths can be focused to a smaller spot permitting the dimension of the channels to be reduced. Two shorter wavelength, high power lasers to which silicon is transparent are the CO laser (wavelength $=5-6 \mu \mathrm{m}$ ) and the Nd: YAG laser (wavelength $=1.06 \mu \mathrm{m}$ ). Also for better resolution, the alignment can be carried out under a microscope with the aid of an infrared converter.

Another significant problem in the PIZM processing is the generation of misfit dislocations due to thermal stress. Implementing active devices is not recommended on a semiconductor surface where dislocation networks are formed. The use of lower eutectic point material and the reduction of the power during processing alleviated this problem, but could not eliminate it.

One conceptual possibility of producing a large number of dislocation-free transsubstrate channels with smaller dimensions is to drill microholes using an etching technique, such as an anisotropic etching [6], dope the sidewalls by an ordinary diffusion techniques, and fill the holes with metal.

\section{ACKNOWLEDGMENT}

Most of the processing work has been done in the National Research and Resource Facility for Submicron Structures (NRRFSS) at Cornell University, Ithaca, NY.

The authors would especially like to thank Prof. E. Wolf and Prof. G. Wolga for the use of the equipment in the facility. The first author also thanks Prof. C. A. Lee of Cornell University and Prof. B. C. Wheeler of the University of Illinois, Urbana-Champaign, for consultation and discussion on various aspects of this work.

\section{REFERENCES}

[1] R. S. Pickard, "A review of printed circuit microelectrodes and their production," J. Neurosci. Methods, vol. 1, pp. 301-318, 1979.

[2] K. D. Wise and J. B. Angell, "A low capacitance multielectrode probe for use in extracellular neurophysiology," IEEE Trans. Biomed. Eng., vol. BME-22, pp. 212-219, 1975.

[3] D. T. Jobling, J. G. Smith, and H. V. Wheal, "Active microelectrode array to record from the mammalian central nervous system in vitro," Med. Biol. Eng. Comput., vol. 19, pp. 553-560, 1981.

[4] L. C. Kimerling, H. J. Leamy, and K. A. Jackson, "Photo induced zone migration (PIZM) in semiconductors," in Proc. Symp. Laser Electron Beam Processing Electron. Mat., vol. 80-1, 1979, pp. $242-$ 248.

[5] T. R. Anthony and H. E. Cline, "Deep-diode arrays," J. Appl. Phys., vol. 47, pp. 2550-2557, 1976.

[6] K. E. Bean, "Anisotropic etching of silicon," IEEE Trans. Electron Devices, vol. ED-25, pp. 1185-1193, 1978.

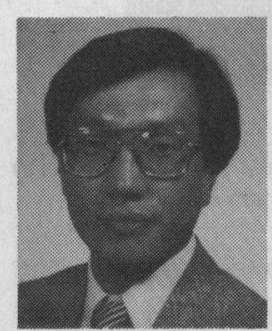

Sung J. Kim (S'80-M'83) was born in Seoul, Korea, on October 24,1954 . He received the B.S. degree in electronics engineering from Seoul National University, Seoul, Korea, in 1978 and the M.S. and Ph.D. degrees in electrical engineering from Cornell University, Ithaca, NY, in 1981 and 1983 , respectively.

At Cornell, he was engaged in developing elec trodes for chornic neural recordings and fabricating microelectrode arrays using semiconductor processing techniques. He is currently a member of the Technical Staff of AT\&T Bell Laboratories, Allentown, PA. His professional interests include neurophysiology, and design and processing of VLSI circuits and devices.

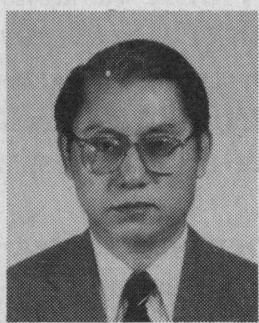

Myunghwan Kim (S'60-M'63-SM'74) was born in Seoul, Korea, on February 8, 1932. He received the B.S. degree in electrical engineering from the University of Alabama, Tuscaloosa, in 1958 and the M.E. and the Ph.D. degrees in electrical engineering from Yale University, New Haven, CT, in 1959 and 1962, respectively.

From 1959 to 1960 he was an Electrical Engineer at the Tennessee Valley Authority, Chattanooga. From 1961 to 1962 he was an Instructor at New Haven College, New Haven, CT. Since 1962 he has been with the School of Electrical Engineering, Cornell University, Ithaca, NY, where he is currently a Professor of Electrical Engineering. He is also an Adjunct Professor of Electrical Engineering at Korea Advanced Institute of Science and Technology, Seoul. His other experiences include: National Research Council Senior Postdoctoral Associate at the Jet Propulsion Laboratory from 1968 to 1969; Visiting Associate at California Institute of Technology, Pasadena, 1969; Visiting Professor at Korea University and Korea Advanced Institute of Science and Technology, Seoul, in 1982.

Dr. Kim is a member of the New York Academy of Sciences. He was a Danforth Fellow at Yale from 1958 to 1962; and he received the National Institutes of Health Special Research Fellowship in 1969-1970.

William J. Heetderks (M'85) received the Ph.D. degree in bioengineering from the University of Michigan, Ann Arbor, in 1975.

He taught in the Department of Electrical Engineering, Cornell University, Ithaca, NY, from 1975 to 1981 . He took a leave of absence from Cornell to attend the University of Miami Ph.D. to M.D. program where he received the M.D. degree in 1983 . He is now completing a residency in internal medicine at Butterworth Hospital, Grand Rapids, MI. 